\title{
THE MONODROMY CONJECTURE FOR ZETA FUNCTIONS ASSOCIATED TO IDEALS IN DIMENSION TWO
}

\author{
Lise Van Proeyen and Willem Veys *
}

\begin{abstract}
The monodromy conjecture states that every pole of the topological (or related) zeta function induces an eigenvalue of monodromy. This conjecture has already been studied a lot. It is proven for zeta functions associated to polynomials in two variables. Many partial results are found for polynomials in three variables.

In this article we work with zeta functions associated to an ideal. First we work in arbitrary dimension and obtain a formula (like the one of A'Campo) to compute the 'Verdier monodromy' eigenvalues associated to an ideal. Afterwards we prove a generalized monodromy conjecture for arbitrary ideals in two variables.
\end{abstract}

2000 Mathematics Subject Classification. 14E15, 32S40, 14H20.

\section{Introduction}

Classically the invariants called topological, motivic and $p$-adic Igusa zeta function are associated to one polynomial $f$ over $\mathbb{C}$, over an arbitrary field of characteristic zero and over a $p$-adic field, respectively. There are fascinating conjectures relating their poles with the roots of the Bernstein-Sato polynomial (also called $b$-function) of $f$ and with the eigenvalues of the local Milnor monodromy of $f$ in points of $\{f=0\}$, up to now proven in full generality only for polynomials in two variables.

One associates similarly in a natural way all these functions to several polynomials or to an ideal. Not so obvious is the notion of Bernstein-Sato polynomials associated to several polynomials or an ideal. There is a construction of Sabbah [Sa] and more recently also of Budur, Mustaţă and Saito [BMS]. Concerning monodromy, the classical construction of local Milnor

${ }^{*}$ K.U.Leuven, Departement Wiskunde, Celestijnenlaan 200B, B-3001 Leuven, Belgium, email: Lise.VanProeyen@wis.kuleuven.be,Wim.Veys@wis.kuleuven.be. The research was partially supported by the Fund of Scientific Research - Flanders (G.0318.06). 
fibre does not generalize to arbitrary maps $\left(f_{1}, \ldots, f_{r}\right)$. However, there is a notion of 'Verdier monodromy' in this general context [Ver].

In this paper we prove a relation between the poles of these zeta functions associated to an arbitrary ideal in two variables and the "Verdier monodromy eigenvalues' of this ideal, generalizing the result for one polynomial.

We now provide more details, focussing on the topological zeta function. Let $\mathcal{I}=\left(f_{1}, \ldots, f_{r}\right)$ be a nontrivial ideal in $\mathbb{C}\left[x_{1}, \ldots, x_{n}\right]$ and $Y=\mathbf{V}(\mathcal{I})$ its associated subscheme of $\mathbb{A}_{\mathbb{C}}^{n}$. We assume that $Y$ contains the origin.

We first fix notation to define the topological zeta function. Take a principalization $\psi: \tilde{X} \rightarrow \mathbb{A}^{n}$ of $\mathcal{I}$. This means that $\psi$ is a proper birational map from a nonsingular variety $\tilde{X}$ such that the total transform $\psi^{*} \mathcal{I}$ is principal and moreover is the ideal of a simple normal crossings divisor. Let $\tilde{E}=\sum_{i \in J} N_{i} E_{i}$ denote this divisor, i.e. its irreducible components are the $E_{i}, i \in J$, occurring with multiplicity $N_{i}$. (Alternatively, one can say that $\psi^{-1} Y=\tilde{E}$.) Let the relative canonical divisor of $\psi$ be $\sum_{i \in J}\left(\nu_{i}-1\right) E_{i}$, i.e. $\nu_{i}-1$ is the multiplicity of $E_{i}$ in the divisor of $\psi^{*}\left(d x_{1} \wedge \ldots \wedge d x_{n}\right)$. Finally put $E_{I}^{\circ}:=\left(\cap_{i \in I} E_{i}\right) \backslash\left(\cup_{l \notin I} E_{l}\right)$ for $I \subset J$; these $E_{I}^{\circ}$ form a natural locally closed stratification of $\tilde{X}$. (Note that $E_{\emptyset}^{\circ}=\tilde{X} \backslash \cup_{l \in J} E_{l}$.) The (local) topological zeta function of $\mathcal{I}$ at 0 is

$$
Z_{t o p, \mathcal{I}}(s):=\sum_{I \subset J} \chi\left(E_{I}^{\circ} \cap \psi^{-1}\{0\}\right) \prod_{i \in I} \frac{1}{\nu_{i}+s N_{i}} \in \mathbb{Q}(s) .
$$

There is a global version replacing $E_{I}^{\circ} \cap \psi^{-1}\{0\}$ by $E_{I}^{\circ}$.

When $r=1$, Denef and Loeser showed in [DL1] that the expression above does not depend on the chosen principalization (which for $r=1$ is just an embedded resolution) by writing it as a limit of $p$-adic Igusa zeta functions. Alternatively, they obtained it later in [DL2] as a specialization of the motivic zeta function. This can be generalized to arbitrary $r$, see e.g. [VZ, (2.4)]. Still another possibility for arbitrary $r$ is to use the Weak Factorization Theorem of Włodarczyk et al. [AKMW] to compare two principalizations. At any rate, observe that a complete list of possible poles of $Z_{t o p, \mathcal{I}}(s)$ is given by the $-\frac{\nu_{i}}{N_{i}}, i \in J$.

For $r=1$, say $\mathcal{I}=(f)$, there are the following intriguing conjectures. (They were originally formulated for the $p$-adic Igusa zeta function, which is a certain $p$-adic integral, and partially motivated by analogous statements that are true for a similar complex integral.)

Conjecture 1. If $s_{0}$ is a pole of $Z_{t o p, f}(s)$, then $s_{0}$ is a root of the (local) Bernstein-Sato polynomial $b_{f, 0}(s)$ of $f$.

Conjecture 2. (Monodromy Conjecture.) If $s_{0}$ is a pole of $Z_{t o p, f}(s)$, then $e^{2 \pi i s_{0}}$ is an eigenvalue of the local monodromy action on some coho- 
mology group of the Milnor fibre of $f$ at some point of $\{f=0\}$ close to 0 .

Note that Conjecture 1 implies Conjecture 2 since for any root $s_{0}$ of $b_{f, 0}(s)$ we have that $e^{2 \pi i s_{0}}$ is such a monodromy eigenvalue $[\mathrm{M}]$. For $n=2$ Conjecture 1 was proved by Loeser [L1]. He also verified it for non-degenerate polynomials satisfying extra assumptions [L2]. (There is a more elementary proof of Conjecture 2 for $n=2$ by Rodrigues [R].) Concerning Conjecture 2, there are various partial results, mainly for $n=3$, by Artal, Cassou-Noguès, Luengo and Melle [ACLM1], [ACLM2], and Lemahieu, Rodrigues and the second author [Vey2], [RV], [Vey3], [LV].

For arbitrary $r$, a Bernstein-Sato polynomial associated to $f_{1}, \ldots, f_{r}$ was constructed by Sabbah in [Sa]. More recently Budur, Mustaţă and Saito introduced a different version, only depending on the ideal $\mathcal{I}=\left(f_{1}, \ldots, f_{r}\right)$. (Their polynomial coincides with a polynomial that appears in [G].)

Still for arbitrary $r$, the notion of local Milnor fibre is in general not well-defined. There is however the following construction of Verdier. To any constructible complex of sheaves $\mathcal{F}^{\bullet}$ on $\mathbb{A}^{n}$, he associates a similar complex on $C_{Y} \mathbb{A}^{n}$, the normal cone of $Y=\mathbf{V}(\mathcal{I})$ in $\mathbb{A}^{n}$. This complex is called the specialization of $\mathcal{F}^{\bullet}$. It is moreover equipped with a 'canonical monodromy operator'. In particular when $\mathcal{I}=(f)$ it turns out that this specialization of $\mathbb{C}^{\bullet}$ is in some sense equivalent to the usual complex of nearby cycles on $Y=\mathbf{V}(f)$, and that the two monodromy notions essentially correspond.

A nice feature of the Bernstein-Sato polynomial of [BMS] is that for any of its roots $s_{0}$, we have that $e^{2 \pi i s_{0}}$ is a 'Verdier monodromy eigenvalue', see [BMS, Corollary 2.8]. This thus generalizes the implication for $r=1$ mentioned above.

It is natural to ask also for arbitrary $\mathcal{I}$ if poles of the topological zeta function of $\mathcal{I}$ are always roots of its Bernstein-Sato polynomial (say the one of $[\mathrm{BMS}]$ ), remembering of course that this question turned out to be very difficult already for $r=1$. In the special case of a monomial ideal $\mathcal{I}$ this was verified in [HMY] by Howald, Mustaţă and Yuen (for the $p$-adic Igusa zeta function). Maybe more accessible, do the poles of the topological zeta function of an arbitrary ideal $\mathcal{I}$ induce monodromy eigenvalues in the sense of Verdier? The main result of this paper is to provide an affirmative answer to the last question for arbitrary ideals in two variables.

The plan of the paper is as follows. In $\S 2$ we recall some facts about 'classical' monodromy in a general context of functions on singular spaces, and in $\S 3$ we explain the construction of the specialization functor of Verdier. In arbitrary dimension we show in $\S 4$ a formula for the 'Verdier monodromy eigenvalues' of an ideal $\mathcal{I}$ in terms of a principalization of the ideal, in the 
same spirit as A'Campo's formula [A] for the eigenvalues of one function $f$ in terms of an embedded resolution of $Y=\mathbf{V}(f)$. Note that this is a priori not obvious; for $r=1$ the complex of nearby cycles lives on $Y$ but for $r>1$ the specialized complex of Verdier lives not on $Y$ but on $C_{Y} \mathbb{A}^{n}$. In $\S 5$ we prove the 'generalized Monodromy Conjecture' for ideals in two variables, and finally in $\S 6$ we provide some examples.

Acknowledgements: The authors want to thank J. Schürmann for the interesting conversations and explanations.

\section{Monodromy}

We start with a function $f: X \rightarrow \mathbb{C}$, where $X$ is a $n$-dimensional complex variety. We put $X_{0}=f^{-1}\{0\}$. To every point $x \in X_{0}$ is associated its local Milnor fibre $F_{x}$ with monodromy actions $M_{x}^{m}: H^{m}\left(F_{x}\right) \rightarrow H^{m}\left(F_{x}\right)$ on the cohomology groups. (We use cohomology with coefficients in $\mathbb{C}$.) Because we are interested in the eigenvalues of the monodromy actions, we put them in a zeta function. The monodromy zeta function of $f$ in a point $x \in X_{0}$ is given by

$$
Z(f, x)(t)=\prod_{m=0, \ldots, n-1} \operatorname{det}\left(I d-t M_{x}^{m}\right)^{(-1)^{m}} .
$$

We can also give another description of this zeta function in terms of sheaf complexes. First, we work with a pair $\left(E^{\bullet}, u\right)$ where $E^{\bullet}$ is a bounded complex of $\mathbb{C}$-vector spaces and $u: E^{\bullet} \rightarrow E^{\bullet}$ an automorphism for which there exists a $N \in \mathbb{Z}_{\geq 0}$ such that $u^{N}=I d$. Note that boundedness is actually a condition on the cohomology vector spaces. For this pair we set

$$
Z\left(E^{\bullet}, u\right)(t)=\prod_{m \in \mathbb{Z}} \operatorname{det}\left(I d-t H\left(u^{m}\right)\right)^{(-1)^{m}},
$$

where $H\left(u^{m}\right): H^{m}\left(E^{\bullet}\right) \rightarrow H^{m}\left(E^{\bullet}\right)$. This is a finite product. When we start with a pair $\left(\mathcal{F}^{\bullet}, u\right)$, where $\mathcal{F}^{\bullet} \in D_{c}^{b}\left(X_{0}\right)$ and $u: \mathcal{F}^{\bullet} \rightarrow \mathcal{F}^{\bullet}$ again an automorphism which satisfies $u^{N}=I d$, we put

$$
Z\left(\mathcal{F}^{\bullet}, u\right)(x)=Z\left(\mathcal{F}_{x}^{\bullet}, u_{x}\right)
$$

for $x \in X_{0}$. (The category $D_{c}^{b}\left(X_{0}\right)$ is the subcategory of the derived category $D\left(X_{0}\right)$ consisting of bounded and constructible complexes of sheaves of $\mathbb{C}$ vector spaces on $X_{0}$. This means that $H^{n}\left(\mathcal{F}^{\bullet}\right)=0$ for $|n|>>0$ and that all the cohomology sheaves are constructible.)

With this notation, we can give an alternative description of the zeta function of monodromy in a point $x \in X_{0}$ :

$$
Z(f, x)=Z\left(\psi_{f} \mathbb{C}_{X}^{\bullet}\right)(x),
$$


where $\psi_{f}$ is the nearby cycle functor of Deligne as defined in [Del], see also [Di, section 4.2]. Moreover, a description of the automorphism on $\psi_{f} \mathbb{C}_{X}^{\bullet}$ (that we didn't explicitly mention above) is also given there. Interesting to remark is that the map $X_{0} \rightarrow \mathbb{C}(t), x \mapsto Z(f, x)$ is a constructible function on $X_{0}$.

The following (easier) formula to work with the monodromy zeta function was found by A'Campo in $[\mathrm{A}]$.

Theorem 2.1. Let $X$ be a variety and $f: X \rightarrow \mathbb{C}$ a function on $X$ for which Sing $(X) \subset f^{-1}\{0\}=X_{0}$. Let $\pi: Y \rightarrow X$ be an embedded resolution of singularities for $X_{0}$. Let $E_{1}, \ldots, E_{s}$ be the smooth irreducible components of $\pi^{-1}\left(X_{0}\right)$. Let $N_{j}$ be the vanishing order of $f \circ \pi$ along $E_{j}$. Then

$$
Z(f, x)(t)=\prod_{j=1}^{s}\left(1-t^{N_{j}}\right)^{\chi\left(E_{j}^{\circ} \cap \pi^{-1}(x)\right)},
$$

where $x \in X_{0}$ and $E_{j}^{\circ}=E_{j} \backslash\left(\cup_{i=1, \ldots, s ; i \neq j} E_{i}\right)$.

This formula was originally proven for functions on $\mathbb{C}^{n}$. But one can check that the proof that Dimca gives in [Di, Corollary 6.1.15] is still valid in this more general context.

\section{The specialization functor of Verdier}

Let $\mathcal{I}$ be an ideal sheaf on a variety $X$. Consider the associated subscheme $Y=\mathbf{V}(\mathcal{I})$ of $X$. We construct the blow-up $\pi: B=B l_{Y} X \rightarrow X$ of $X$ in $Y$ and denote by $E$ the inverse image $\pi^{-1}(Y)$.

For every $e \in E$ we want to study the zeta function of monodromy of the ideal $\mathcal{I}$. To define what monodromy is in this context, we will need notions as the normal cone of $Y$ in $X$ and the specialization functor of Verdier.

The normal cone of $Y$ in $X$ is a cone over $Y$, whose exact definition is given by

$$
C_{Y} X=\operatorname{Spec}\left(\oplus_{n \geq 0} \mathcal{I}^{n} / \mathcal{I}^{n+1}\right)
$$

and can be found in [F, B.6]. Interesting to notice is that the projectivization $P\left(C_{Y} X\right)$ of the normal cone is exactly the exceptional variety $E$ of the blowing-up of $X$ in $Y$. We can identify the 'locus of vertices' of this cone with $Y$. So we have an embedding $j: Y \rightarrow C_{Y} X$ and a projection $p: C_{Y} X \rightarrow Y$. These maps satisfy $p \circ j=I d_{Y}$. Moreover, we have an action of $\mathbb{C}^{*}$ on the normal cone, coming from the graduation on $\oplus_{n \geq 0} \mathcal{I}^{n} / \mathcal{I}^{n+1}$. The locus of vertices is the scheme of the fixed points of the action and the morphisms $j$ and $p$ commute with it. Starting with a point $e \in\left(C_{Y} X\right) \backslash Y$, we define the ruler through $e$ as the orbit of $e$ under the action of $\mathbb{C}^{*}$, so every ruler can be identified with $\mathbb{C}^{*}$. 
The construction of the normal cone is functorial in the following sense. Suppose we have a map $f: X^{\prime} \rightarrow X$ of schemes and two subschemes $Y \subset X$ and $Y^{\prime} \subset X^{\prime}$ such that

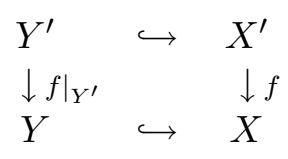

is a cartesian diagram, then we can associate a map $C(f): C_{Y^{\prime}} X^{\prime} \rightarrow C_{Y} X$ to $f$. In particular, the map $\pi: B \rightarrow X$ has an associated map $C(\pi)$ : $C_{E} B \rightarrow C_{Y} X$. This last one has the following interesting properties which we will use further on. If we restrict the map $C(\pi)$ to the 'punctured' cones, $C^{\prime}(\pi):\left(C_{E} B\right) \backslash E \rightarrow\left(C_{Y} X\right) \backslash Y$, this is an isomorphism. Likewise, the map of projectivized cones $C^{\prime \prime}(\pi): P\left(C_{E} B\right) \rightarrow P\left(C_{Y} X\right)$ is well-defined and is an isomorphism. Moreover, we have the following commutative diagram.

$$
\begin{array}{lllll}
E & \longleftarrow & P\left(C_{E} B\right) & \longleftarrow & \left(C_{E} B\right) \backslash E \\
\downarrow \pi & \circ & \downarrow C^{\prime \prime}(\pi) & \circ & \downarrow C^{\prime}(\pi) \\
Y & \longleftarrow & P\left(C_{Y} X\right) & \longleftarrow & \left(C_{Y} X\right) \backslash Y
\end{array}
$$

The deformation to the normal cone of $Y$ in $X$ is a scheme $\hat{X}$ with a morphism $\hat{\pi}: \hat{X} \rightarrow \mathbb{A}^{1}$ such that $\hat{\pi}^{-1}\left(\mathbb{A}^{1} \backslash\{0\}\right) \cong X \times\left(\mathbb{A}^{1} \backslash\{0\}\right)$ and the fibre over $0 \in \mathbb{A}^{1}$ is the normal cone $C_{Y} X$. So we obtain the following commutative diagram.

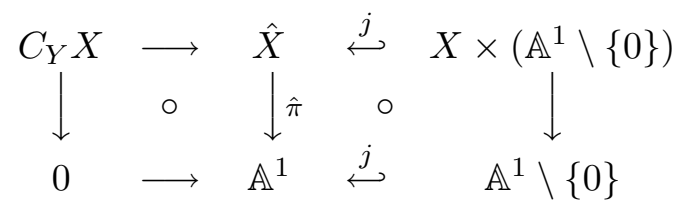

If we have a constructible complex of sheaves $\mathcal{F}^{\bullet}$ on $X$, we can use the specialization functor of Verdier to associate a constructible complex of sheaves on $C_{Y} X$ to it. As in [Ver], we set

$$
S p_{Y \backslash X}\left(\mathcal{F}^{\bullet}\right):=\psi_{\hat{\pi}}\left(j ! p r_{1}^{*} \mathcal{F}^{\bullet}\right)
$$

where $\hat{\pi}$ and $j$ are as before, $p r_{1}$ is the projection of $X \times\left(\mathbb{A}^{1} \backslash\{0\}\right)$ on the first factor and $\psi_{\hat{\pi}}$ is the nearby cycle functor of Deligne. In [Ver, p.356-357], Verdier defines a canonical transformation of monodromy on the complex of sheaves $S p_{Y \backslash X}\left(\mathbb{C}_{X}^{\bullet}\right)$.

This specialization functor has a number of important properties, from which we will give the ones that we need in this article. These properties are stated in [Ver, sections 8 and 10]. 
(SP1) Monodromy. For every constructible complex of sheaves $\mathcal{F}^{\bullet}$, the complex $S p_{Y \backslash X}\left(\mathcal{F}^{\bullet}\right)$ is monodromic. This means that $S p_{Y \backslash X}\left(\mathcal{F}^{\bullet}\right)$ is locally constant on every ruler of $C_{Y} X$.

(SP2) Proper direct image. Suppose we have a cartesian diagram

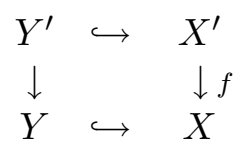

where $f$ is proper. Let $C(f): C_{Y^{\prime}} X^{\prime} \rightarrow C_{Y} X$ be the morphism associated to $f$. Then $C(f)$ is proper and for each constructible complex $\mathcal{F}^{\bullet}$ on $X^{\prime}$, the natural morphism

$$
S p_{Y \backslash X}\left(R f_{*} \mathcal{F}^{\bullet}\right) \rightarrow R C(f)_{*}\left(S p_{Y^{\prime} \backslash X^{\prime}}\left(\mathcal{F}^{\bullet}\right)\right)
$$

is an isomorphism.

(SP6) Normalization. Suppose that $Y$ is a principal divisor with equation $f=0$. Then the morphism $p \times C(f): C_{Y} X \rightarrow Y \times \mathbb{A}^{1}$ is an isomorphism, where $p: C_{Y} X \rightarrow Y$ is the projection. Notice that $C_{\{0\}} \mathbb{A}^{1}=\mathbb{A}^{1}$, so $C(f)$ is indeed a map $C_{Y} X \rightarrow \mathbb{A}^{1}$. With this isomorphism, we can define a section for $z \in \mathbb{C} \backslash\{0\}$ :

$$
\begin{aligned}
s_{z}: Y & \rightarrow C_{Y} X \\
y & \mapsto(p \times C(f))^{-1}(y, z) .
\end{aligned}
$$

Then we have for each constructible complex of sheaves $\mathcal{F}^{\bullet}$ on $X$ an isomorphism

$$
s_{z}^{*}\left(S p_{Y \backslash X}\left(\mathcal{F}^{\bullet}\right)\right) \rightarrow \psi_{f}\left(\mathcal{F}^{\bullet}\right) .
$$

This isomorphism is compatible with the two monodromy operations defined on both sheaves on $Y$, in the sense that they are each others opposite.

(SP7) Perversity. The specialization functor transforms perverse sheaves into perverse sheaves.

\section{The zeta function of monodromy}

As we said before, Verdier defined a canonical transformation of monodromy on the complex of sheaves $S p_{Y \backslash X}\left(\mathbb{C}_{X}^{\bullet}\right)$, which we will denote by $M$. For each $m \in \mathbb{Z}$ and $y \in\left(C_{Y} X\right) \backslash Y$, we have an automorphism $M_{y}^{m}$ on the stalk $S p_{Y \backslash X}^{m}\left(\mathbb{C}_{X}^{\bullet}\right)_{y}$. Eigenvalues of monodromy are eigenvalues of these vector space automorphisms. 
The zeta function of monodromy for a point $e \in E$ is defined as

$$
Z_{\mathcal{I}, e}(t)=Z\left(S p_{Y \backslash X}\left(\mathbb{C}_{X}^{\bullet}\right), M\right)\left(e^{\prime}\right),
$$

where $e^{\prime}$ is an arbitrary point on $\left(C_{Y} X\right) \backslash Y$ that is mapped to $e$ by the projectivization. We use the definition of $Z(\cdot)$ given in section 2. Note that, by (SP1), this definition is independent of the choice of $e^{\prime}$.

In the next section, we use the zeta function of monodromy to prove the monodromy conjecture. We will prove that a pole $s_{0}$ of the topological zeta function induces a zero or a pole $e^{2 \pi i s_{0}}$ of the monodromy zeta function for some point $e \in E$. This implies that this number $e^{2 \pi i s_{0}}$ is an eigenvalue of monodromy.

Remark 4.1. Note that it is also true that each eigenvalue of monodromy is a zero or a pole of the monodromy zeta function for some point $e \in E$. Since we know from (SP7) that $S p_{Y \backslash X}\left(\mathbb{C}_{X}^{\bullet}\right)$ is a perverse sheaf, we can copy the proof of [Den, Lemma 4.6] to obtain this result.

Now we prove a generalization of the formula of A'Campo (Theorem 2.1) to the case of ideals.

Theorem 4.2. Let $\mathcal{I}$ be a sheaf of ideals on a variety $X$. Let $Y=\mathbf{V}(\mathcal{I})$ be the associated subscheme of $X$ and suppose that $\operatorname{Sing}(X) \subset Y$. Let $\pi: B=$ $B l_{Y} X \rightarrow X$ be the blow-up of $X$ in $Y$ and $\psi: \tilde{X} \rightarrow X$ a principalization of $\mathcal{I}$. Define $\varphi: \tilde{X} \rightarrow B$ as the unique morphism such that $\psi=\pi \circ \varphi$. We denote by $E$ the inverse image $\pi^{-1}(Y)$ and $\tilde{E}=\psi^{-1}(Y)$. We use $E_{i}, i \in J$, for the irreducible components of $\tilde{E}$ and $N_{i}$ for the according multiplicities in $\tilde{E}$. Put $E_{i}^{\circ}=E_{i} \backslash \cup_{j \in J, j \neq i} E_{j}$. For a point $e \in E$, the zeta function of monodromy is equal to

$$
Z_{\mathcal{I}, e}(t)=\prod_{j \in J}\left(1-t^{N_{j}}\right)^{\chi\left(E_{j}^{\circ} \cap \varphi^{-1}(e)\right)} .
$$

Remark 4.3. Note that the principalization $\psi$ factorizes through the blow-up $\pi$ since the inverse image $\psi^{-1}(Y)$ is a Cartier subscheme in $\tilde{X}$.

Proof. Fix $e \in E$ and choose an element $e^{\prime} \in\left(C_{Y} X\right) \backslash Y$ that is mapped to $e$ by the projectivization map.

The restriction of $\pi$ is an isomorphism $B \backslash E \rightarrow X \backslash Y$, which implies that $\left.R \pi_{*} \mathbb{C}_{B}^{\bullet}\right|_{X \backslash Y}=\left.\mathbb{C}_{X}^{\bullet}\right|_{X \backslash Y}$. Since $\left.S p_{Y \backslash X}\left(\mathcal{F}^{\bullet}\right)\right|_{\left(C_{Y} X\right) \backslash Y}$ only depends on $\left.\mathcal{F}\right|_{X \backslash Y}$ (see [Ver, p354]), we can deduce that

$$
\left.S p_{Y \backslash X}\left(\mathbb{C}_{X}^{\bullet}\right)\right|_{\left(C_{Y} X\right) \backslash Y}=\left.S p_{Y \backslash X}\left(R \pi_{*} \mathbb{C}_{B}^{\bullet}\right)\right|_{\left(C_{Y} X\right) \backslash Y}
$$

and

$$
Z\left(S p_{Y \backslash X}\left(\mathbb{C}_{X}^{\bullet}\right)\right)\left(e^{\prime}\right)=Z\left(S p_{Y \backslash X}\left(R \pi_{*} \mathbb{C}_{B}^{\bullet}\right)\right)\left(e^{\prime}\right)
$$


Because we have the cartesian diagram

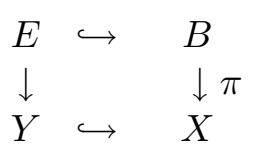

we can use (SP2) to write that

$$
S p_{Y \backslash X}\left(R \pi_{*} \mathbb{C}_{B}^{\bullet}\right) \stackrel{\sim}{\longrightarrow} R C(\pi)_{*}\left(S p_{E \backslash B}\left(\mathbb{C}_{B}^{\bullet}\right)\right)
$$

is an isomorphism, or that

$$
Z\left(S p_{Y \backslash X}\left(R \pi_{*} \mathbb{C}_{B}^{\bullet}\right)\right)\left(e^{\prime}\right)=Z\left(S p_{E \backslash B}\left(\mathbb{C}_{B}^{\bullet}\right)\right)\left(C(\pi)^{-1}\left(e^{\prime}\right)\right) .
$$

(Note that the restriction of $C(\pi)$ to $\left(C_{E} B\right) \backslash E$ is an isomorphism, as said in $\S 3$.)

There exists an open $V_{e}$ around $e$ such that $E \cap V_{e}$ is the zero locus of one nonzerodivisor $f$. So we can use (SP6) to see that locally, we have an isomorphism $p \times C(f): C_{E} B \rightarrow E \times \mathbb{A}^{1}$. Take $z \in \mathbb{C} \backslash\{0\}$ such that $(p \times C(f))\left(C(\pi)^{-1}\left(e^{\prime}\right)\right)=(e, z)$. This is possible, using the commutative diagram (3.1). We can conclude that we have an isomorphism

$$
s_{z}^{*}\left(S p_{E \backslash B}\left(\mathbb{C}_{B}^{\bullet}\right)\right) \longrightarrow \psi_{f}\left(\mathbb{C}_{B}^{\bullet}\right) .
$$

This isomorphism is 'compatible' with the monodromy actions, so

$$
Z\left(S p_{E \backslash B}\left(\mathbb{C}_{B}^{\bullet}\right)\right)\left(s_{z}(e)\right)=Z\left(\psi_{f}\left(\mathbb{C}_{B}^{\bullet}\right)\right)(e) .
$$

The right hand side is the monodromy zeta function of the map $f: V_{e} \subset$ $B \rightarrow \mathbb{C}$ in the point $e$. Because we already have an embedded resolution of $E$ in $B$, namely the map $\varphi: \tilde{X} \rightarrow B$, we can easily use the formula of A'Campo (Theorem 2.1) to see that

$$
Z\left(\psi_{f}\left(\mathbb{C}_{B}^{\bullet}\right)\right)(e)=\prod_{j \in J}\left(1-t^{N_{j}}\right)^{\chi\left(E_{j}^{\circ} \cap \varphi^{-1}(e)\right)} .
$$

For this last equality, we need that the multiplicities of the irreducible components in the exceptional locus of the principalization, are the same as these in the exceptional locus of the resolution of singularities. This is true, what we can see in

$$
\tilde{E}=\sum_{i \in J} N_{i} E_{i}=(\pi \circ \varphi)^{-1}(Y)=\varphi^{-1}\left(\pi^{-1} Y\right)=\varphi^{-1}(E) .
$$

Putting all these equalities of zeta functions together, proves our theorem. 


\section{The Monodromy Conjecture}

From now on, we will work in dimension 2, but we will use the same notation as before. So let $\mathcal{I} \subset \mathbb{C}[x, y]$ be an ideal satisfying $\{0\} \subset \operatorname{Supp} \mathcal{I}$. Put $Y=$ $V(\mathcal{I})$ the subscheme of $X=\mathbb{C}^{2}$ defined by $\mathcal{I}$. Let $\pi: B=B l_{Y} X \rightarrow X$ be the blowing-up of $X$ in $Y$ and $\psi: \tilde{X} \rightarrow X$ be the minimal principalization of $\mathcal{I}$. The map $\varphi: \tilde{X} \rightarrow B$ is defined such that $\psi=\pi \circ \varphi$. The exceptional locus in $B$ is $E=\pi^{-1}(Y)$. The exceptional locus in $\tilde{X}$ is $\tilde{E}=\psi^{-1}(Y)=\sum_{i \in J} N_{i} E_{i}$, where $E_{i}\left(N_{i}, \nu_{i}\right)$ are the irreducible components of $\tilde{E}$, together with their numerical data.

Theorem 5.1. (Generalized Monodromy Conjecture.) If $-\frac{\nu}{N}$ is a pole of the local topological zeta function of an ideal $\mathcal{I} \subset \mathbb{C}[x, y]$, then there exists a point $y \in E$ such that $e^{-2 \pi i \frac{\nu}{N}}$ is an eigenvalue of monodromy in $y$.

We first mention some results that will be useful in the proof.

For an ideal $\mathcal{I}=\left(f_{1}, \ldots, f_{r}\right) \subset \mathbb{C}[x, y]$ we can look at the linear system $\left\{\lambda_{1} f_{1}+\ldots+\lambda_{r} f_{r}=0 \mid \lambda_{i} \in \mathbb{C}\right.$ for $\left.i=1, \ldots, r\right\}$. A total generic curve of $\mathcal{I}$ is a general element of this linear system. Now we determine whether there are common components among the $f_{i}$ and put them together. So we can write $\mathcal{I}=(h)\left(f_{1}^{\prime}, \ldots, f_{r}^{\prime}\right)$, where $\left(f_{1}^{\prime}, \ldots, f_{r}^{\prime}\right)$ is a finitely supported ideal. A generic curve of the ideal $\mathcal{I}$ is a general element of the linear system $\left\{\lambda_{1} f_{1}^{\prime}+\ldots+\lambda_{r} f_{r}^{\prime}=0 \mid \lambda_{i} \in \mathbb{C}\right.$ for $\left.i=1, \ldots, r\right\}$. Notice that the definition of the (total) generic curve is dependent on the choice of generators we use to represent the ideal.

Lemma 5.2. If an irreducible component $E_{i}$ of $\tilde{E}$ is contracted by $\varphi$, then there is no intersection between $E_{i}$ and the strict transform of a generic curve of the ideal $\mathcal{I}$ in $\tilde{X}$.

Proof. If there is an intersection, the strict transform of a generic curve of $\mathcal{I}$ in $B$ contains the point $\varphi\left(E_{i}\right)$. But if we denote $\widetilde{f}_{i}$ for the strict transform of $f_{i}^{\prime}$ in $B$, the linear system $\left\{\lambda_{1} \widetilde{f}_{1}+\ldots+\lambda_{r} \widetilde{f}_{r}=0 \mid \lambda_{i} \in \mathbb{C}\right.$ for $\left.i=1, \ldots, r\right\}$ should be base point free (e.g. see [H, Example 7.17.3]).

Lemma 5.3. Let $\left(f_{1}, \ldots, f_{l}\right)$ be an ideal in $\mathbb{C}[x, y]$ and $E_{0}(N, \nu)$ an exceptional curve of the principalization (together with the numerical data). Suppose $E_{0}$ intersects $n$ times the strict transform of a generic curve and $m$ times other components $E_{1}\left(N_{1}, \nu_{1}\right), \ldots, E_{m}\left(N_{m}, \nu_{m}\right)$ of the principalization. Put $\alpha_{i}=\nu_{i}-\frac{\nu}{N} N_{i}$ for $i=1, \ldots, m$. Then

$$
\sum_{i=1}^{m} \alpha_{i}=m-2+\frac{\nu n}{N}
$$


This equality can be found in [VV, §3]. It is a reformulation of the relation between the numerical data proved by Loeser in [L1].

In [VV, Theorem 4.2], we gave a complete list of five conditions in which a candidate-pole is indeed a pole of the local topological zeta function of an ideal in dimension two. If you use Lemma 5.3, it is not difficult to see that the conditions on the numerical data that are given in numbers 3 and 4 , are equivalent to the condition of being intersected by the strict transform of a generic curve. So we can reformulate this theorem as follows.

Theorem 5.4. Let $\mathcal{I} \subset \mathbb{C}[x, y]$ be an ideal satisfying $0 \in \operatorname{Supp}(\mathcal{I})$ and $\psi: \tilde{X} \rightarrow \mathbb{C}^{2}$ the minimal principalization of $\mathcal{I}$ in a neighbourhood of 0 . Let $E_{\bullet}\left(N_{\bullet}, \nu_{\bullet}\right)$ be the components of the support of the total transform $\psi^{*} \mathcal{I}$ with their associated numerical data.

The rational number $s_{0}$ is a pole of the local topological zeta function of $\mathcal{I}$ if and only if at least one of the following conditions is satisfied:

1. $s_{0}=-\frac{1}{N}$ for a component $E_{0}(N, \nu)$ of the support of the weak transform of $\mathcal{I}$;

2. $s_{0}=-\frac{\nu}{N}$ for $E_{0}(N, \nu)$ an exceptional curve that has non-empty intersection with the strict transform of a generic curve of the ideal $\mathcal{I}$;

3. $s_{0}=-\frac{\nu}{N}$ for $E_{0}(N, \nu)$ an exceptional curve that intersects at least three times other components.

Now we are ready to prove the monodromy conjecture for ideals in dimension 2. We were inspired by $[R]$, where Rodrigues gives an elementary proof of the monodromy conjecture for curves on normal surfaces.

Proof of Theorem 5.1. Choose a pole $s_{0}$ of the local topological zeta function of the ideal $\mathcal{I}$ and take $a, d \in \mathbb{Z}$ satisfying $s_{0}=-\frac{a}{d}$ and $\operatorname{gcd}(a, d)=1$.

Suppose that there exists an irreducible component $E_{m}\left(N_{m}, \nu_{m}\right)$ with $d \mid N_{m}$ that is not contracted to a point by $\varphi: \tilde{X} \rightarrow B$.

Choose a point $y \in \varphi\left(E_{m}^{\circ}\right)$ such that $\varphi^{-1}(y)$ is a finite set of points. Then there exists for every $j \in J$ a nonnegative integer $k_{j}\left(\right.$ with $k_{m} \neq 0$ ) such that

$$
Z_{\mathcal{I}, y}(t)=\prod_{j \in J}\left(1-t^{N_{j}}\right)^{k_{j}},
$$

from which we see that $e^{2 \pi i s_{0}}$ is a zero of the zeta function of monodromy in $y$, so it is an eigenvalue of monodromy.

On the other hand, suppose that every $E_{i}\left(N_{i}, \nu_{i}\right)$ that satisfies $d \mid N_{i}$ is contracted to a point by $\varphi$. This implies (by using Lemma 5.2 and Theorem 5.4) that there exists an integer $m \in J$, with $s_{0}=-\frac{\nu_{m}}{N_{m}}$, such that $E_{m}$ is exceptional and $E_{m}$ intersects at least three times other components of $\tilde{E}$. 
Fix such a $m$ and take $y=\varphi\left(E_{m}\right)$. Define $T \subset J$ such that $\varphi^{-1}\{y\}=\cup_{i \in T} E_{i}$. To prove that $e^{2 \pi i s_{0}}$ is a zero or a pole of the zeta function of monodromy in $y$, it is enough to show that

$$
\sum_{i \in T, d \mid N_{i}} \chi\left(E_{i}^{\circ}\right)<0
$$

First notice that the strict transform of a generic curve intersects at least one exceptional component, so not every exceptional curve can be contracted and it is impossible that $d$ divides $N_{i}$ for every $i \in J$.

We define

$$
\tilde{M}:=\bigcup_{i \in T, d \mid N_{i}} E_{i} .
$$

Every connected component of $\tilde{M}$ contains at least one irreducible component that intersects a component $E_{k}\left(N_{k}, \nu_{k}\right)$ with $d \nmid N_{k}$. (Note that if $d \mid N_{k}$ and $E_{k}$ has a non-empty intersection with $\tilde{M}$, the integer $k$ has to belong to T.) This irreducible component of $\tilde{M}$ also has to intersect a second component $E_{k^{\prime}}\left(N_{k^{\prime}}, \nu_{k^{\prime}}\right)$ with $d \nmid N_{k^{\prime}}$. We can deduce this from the formula $\kappa N_{l}=\sum_{i=1}^{r} N_{i}$, where $E_{l}$ is an exceptional curve intersecting $r$ times other components $E_{i}$, for $i=1, \ldots, r$, and where $-\kappa$ denotes the self-intersection number of $E_{l}$.

Every connected component $\bigcup_{i \in I_{0}} E_{i}$ of $\tilde{M}$ satisfies

$$
\sum_{i \in I_{0}} \chi\left(E_{i}^{\circ}\right) \leq 0
$$

Because such a component has at least two external intersections, this is a direct consequence of the fact that $\sum_{i=1}^{r} \chi\left(E_{i}^{\circ}\right)=2$ when $\bigcup_{i=1}^{r} E_{i}$ is a tree consisting of rational curves (see e.g. [R, Lemma 2.2]).

Now fix the connected component $M=\bigcup_{i \in I_{M}} E_{i}$ of $\tilde{M}$ that contains $E_{m}$. For this one, we prove the strict inequality

$$
\sum_{i \in I_{M}} \chi\left(E_{i}^{\circ}\right)<0
$$

If this sum would equal 0 , then $M$ is a tree of rational curves that intersects precisely two times with components $E_{j}$ outside $\tilde{M}$. From a previous argument, we know that these intersections are on one curve $E_{s}$ with $s \in I_{M}$. There are two possibilities.

$E_{s}=E_{m}$. The component $E_{m}$ will intersect at least one other curve $E_{j}\left(N_{j}, \nu_{j}\right)$. This curve belongs to $M$, so $d \mid N_{j}$ and the number $\nu_{j}-\frac{a}{d} N_{j}$ is an integer. The only possibility is 0 (see [VV, Proposition 3.1]), so this implies that $-\frac{\nu_{j}}{N_{j}}=-\frac{a}{d}$. We know that $E_{j}$ is contracted to a point, so it is not intersected by the strict transform of a generic curve. From 
the relations between the numerical data (see Lemma 5.3), we can deduce that $E_{j}$ intersects at least one other component of $M$ and we can repeat this process infinitely many times. This leads to a contradiction.

$E_{s} \neq E_{m}$. Now we can start for each of the (at least three) components that intersect $E_{m}$ the same procedure of constructing a series of curves in $M$. Only one of these can eventually stop, because there is only one of these series that has intersections outside of $M$.

This ends the proof.

Remark 5.5. Theorem 5.1 is also true for the motivic and the Hodge zeta function of $\mathcal{I}$, and for $p$-adic Igusa zeta functions associated to several polynomials in two variables, because the necessary condition of [VV, Theorem $4.2]$ to be a pole is still valid for these zeta functions. We refer to [VV, §6] for definitions and more explanation.

\section{Some examples}

EXAMPLE 1: $\left(x^{3} y, x^{6}+y^{4}\right)$

As a first example, we will take the ideal $\mathcal{I}=\left(x^{3} y, x^{6}+y^{4}\right) \subset \mathbb{C}[x, y]$ and $Y=\mathbf{V}(\mathcal{I})$. The intersection diagram of the principalization $\tilde{X}$ is as in Figure 1, where the dashed line denotes the strict transform of a generic curve.

Figure 1: Intersection diagram of the principalization of $\left(x^{3} y, x^{6}+y^{4}\right)$.

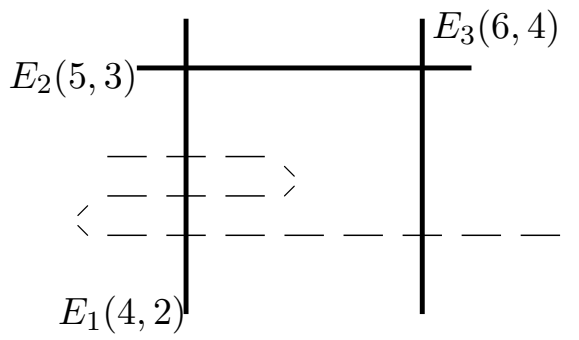

We see that the poles of the local topological zeta function are $-\frac{1}{2}$ and $-\frac{2}{3}$.

The blowing-up $B=B l_{Y} \mathbb{C}^{2}$ is given by

$$
\operatorname{Proj} \frac{\mathbb{C}[x, y][A, B]}{\left(A x^{3} y-B\left(x^{6}+y^{4}\right)\right)},
$$

see e.g. [EH, Section IV.2.1]. The exceptional variety consists of only one irreducible curve, say $E$. 
The map $\varphi: \tilde{X} \rightarrow B$ contracts $E_{2}$ to a point $a$ on $E$. It maps $E_{1}$ and $E_{3}$ surjectively on $E$. The restriction to $E_{3}$ is one-to-one, but the restriction to $E_{1}$ is three-to-one.

If we choose a point $e$ on $E$, different from $a$, we can see that the monodromy zeta function is

$$
Z_{\mathcal{I}, e}(t)=\left(1-t^{4}\right)^{3}\left(1-t^{6}\right) .
$$

We can immediately check that $e^{-\pi i}$ and $e^{-\frac{4 \pi i}{3}}$ are zeroes of this function. Since $-\frac{1}{2}$ and $-\frac{2}{3}$ were the only poles of the local topological zeta function, this illustrates the monodromy conjecture.

ExAMPLE 2: $\left(x^{4}, x y^{2}, y^{3}\right)$

For this example, we will work with the ideal $\mathcal{I}=\left(x^{4}, x y^{2}, y^{3}\right) \subset \mathbb{C}[x, y]$ and $Y=\mathbf{V}(\mathcal{I})$. The intersection diagram of the principalization (with numerical data) is now given in Figure 2.

Figure 2: Intersection diagram of the principalization of $\left(x^{4}, x y^{2}, y^{3}\right)$.

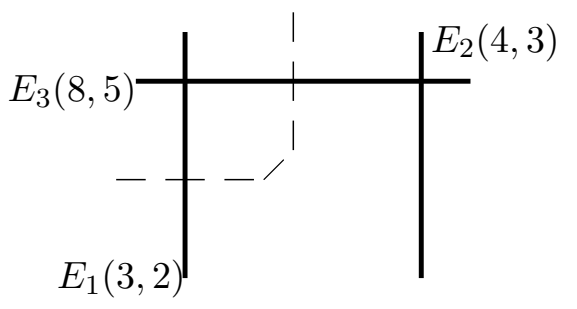

We can easily deduce that the poles of $Z_{t o p, \mathcal{I}}(s)$ are $-\frac{2}{3}$ and $-\frac{5}{8}$.

This time the blowing-up $B=B l_{Y} \mathbb{A}^{2}$ is given by

$$
B=\operatorname{Proj} \frac{\mathbb{C}[x, y][A, B, C]}{\left(y^{2} A+x^{3} B, x C+y B, B^{3} x+A C^{2}\right)}
$$

and the exceptional variety $E$ consists of two projective lines $E^{\prime}$ and $E^{\prime \prime}$.

The map $\varphi: \tilde{X} \rightarrow B$ maps $E_{1}$ and $E_{3}$ one-to-one onto $E^{\prime}$ and $E^{\prime \prime}$, respectively. It contracts $E_{2}$ to a point $a$ on $E^{\prime \prime}$.

We choose a point $x \in E^{\prime \circ}$ and a point $y \in E^{\prime \prime \prime} \backslash\{a\}$. For these points the monodromy zeta functions are

$$
Z_{\mathcal{I}, x}(t)=1-t^{3}, \quad Z_{\mathcal{I}, y}(t)=1-t^{8} .
$$

So we conclude that the two poles of the topological zeta function induce two eigenvalues of monodromy $e^{-\frac{4 \pi i}{3}}$ and $e^{-\frac{5 \pi i}{4}}$. 
Remark 6.1. Note that this example is a monomial ideal. As mentioned in the introduction, the monodromy conjecture had already implicitly been verified in this case.

EXAMPLE 3: $\left(x^{3} y, x^{3}-y^{2}\right)$

In this last example, we set $\mathcal{I}=\left(x^{3} y, x^{3}-y^{2}\right) \subset \mathbb{C}[x, y]$ and again $Y=\mathbf{V}(\mathcal{I})$.

Figure 3: Intersection diagram of the principalization of $\left(x^{3} y, x^{3}-y^{2}\right)$.

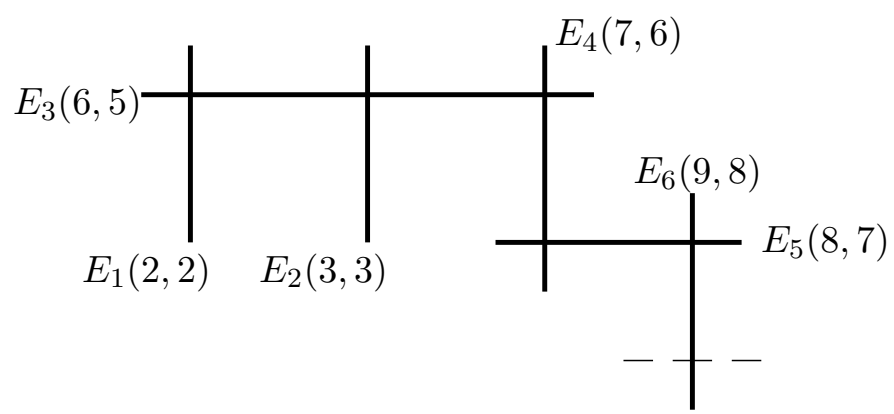

In Figure 3 we can see that $Z_{t o p, \mathcal{I}}(s)$ has poles in $-\frac{5}{6}$ and $-\frac{8}{9}$.

We know that

$$
B=B l_{Y} X=\operatorname{Proj} \frac{\mathbb{C}[x, y][A, B]}{\left(A\left(x^{3}-y^{2}\right)-B x^{3} y\right)} .
$$

The blowing-up has one irreducible exceptional component $E$. The map $\varphi$ : $\tilde{X} \rightarrow B$ maps $E_{6}$ surjectively on $E$ and all the other exceptional components of the principalization are contracted to a point $a$ on $E$. We fix a point $x \in E^{\circ}, x \neq a$, and look at the following two monodromy zeta functions:

$$
Z_{\mathcal{I}, a}(t)=\frac{\left(1-t^{2}\right)\left(1-t^{3}\right)}{1-t^{6}}, \quad Z_{\mathcal{I}, x}(t)=1-t^{9} .
$$

We can see that the two poles of the topological zeta function $-\frac{5}{6}$ and $-\frac{8}{9}$ give rise to eigenvalues of monodromy $e^{-\frac{5 \pi i}{3}}$ and $e^{-\frac{16 \pi i}{9}}$.

\section{References}

[A] N. A'Campo, La fonction zêta d'une monodromie, Comment. Math. Helv. 50 (1975), 233-248.

[ACLM1] E. Artal-Bartolo, P. Cassou-Noguès, I. Luengo and A. Melle-Hernandez, Monodromy conjecture for some surface singularities, Ann. Scient. Ec. Norm. Sup. 35 (2002), 605-640.

[ACLM2] E. Artal Bartolo, P. Cassou-Noguès, I. Luengo and A. Melle-Hernandez, Quasiordinary power series and their zeta functions, Mem. Amer. Math. Soc., vol. 178 , no. 841 (2005). 
[AKMW] D. Abramovich, K. Karu, K. Matsuki, J. Włodarczyk, Torification and factorization of birational maps, J. Amer. Math. Soc. 15 (2002), 531-572.

[BMS] N. Budur, M. Mustaţă and M. Saito, Bernstein-Sato polynomials of arbitrary varieties, Compos. Math. 142 (2006), no. 3, 779-797.

[Del] P. Deligne, Le formalisme des cycles évanescents, in SGA7 XIII and XIV, Lect. Notes in Math. vol. 340, Springer Berlin (1973), 82-115 and 116-164.

[Den] J. Denef, Degree of local zeta functions and monodromy, Compos. Math. 89 (1994), 207-216.

[DL1] J. Denef and F. Loeser, Caractéristiques d' Euler-Poincaré, fonctions zêta locales, et modifications analytiques, J. Amer. Math. Soc. 5 (1992), 705-720.

[DL2] J. Denef and F. Loeser, Motivic Igusa zeta functions, Journal of Algebraic Geometry 7 (1998), 505-537.

[Di] A. Dimca, Sheaves in topology, Springer-Verlag Berlin Heidelberg (2004).

[EH] D. Eisenbud and J. Harris, The Geometry of Schemes, Springer-Verlag New York (2000).

[F] W. Fulton, Intersection Theory, Springer-Verlag Berlin Heidelberg (1984).

[G] A. Gyoja, Bernstein-Sato's polynomial for several analytic functions, J. Math. Kyoto Univ. 33 (1993), 399-411.

[H] R. Hartshorne, Algebraic Geometry, Springer-Verlag New York (1977).

[HMY] J. Howald, M. Mustaţă, C. Yuen, On Igusa zeta functions of monomial ideals, Proc. Amer. Math. Soc. Vol. 135, nr. 11 (2007), 3425-3433.

[L1] F. Loeser, Fonctions d'Igusa p-adiques et polynômes de Bernstein, Amer. J. Math. 110 (1988), 1-21.

[L2] F. Loeser, Fonctions d'Igusa p-adiques, polynômes de Bernstein, et polyèdres de Newton, J. Reine Angew. Math. 412 (1990), 75-96.

[LV] A. Lemahieu and W. Veys, On monodromy for a class of surfaces C. R. Acad. Sci. Paris, 345/11 (2007), 633-638.

[M] B. Malgrange, Polynôme de Bernstein-Sato et cohomologie évanescente, Analysis and topology on singular spaces, II, III (Luminy, 1981), Astérisque 101-102 (1983), 243-267.

[R] B. Rodrigues, On the Monodromy Conjecture for curves on normal surfaces, Math. Proc. of the Cambridge Philosophical Society 136 (2004), 313-324.

[RV] B. Rodrigues and W. Veys, Holomorphy of Igusa's and topological zeta functions for homogeneous polynomials, Pacific J. Math. 201 (2001), 429-441.

[Sa] C. Sabbah, Proximité évanescante, I. La structure polaire d'un D-module, Compos. Math. 62 (1987), 283-328; II. Equations fonctionnelles pour plusieurs fonctions analytiques, ibid. 64 (1987), 213-241.

[Sc] J. Schürmann, A generalized Verdier-type Riemann-Roch theorem for ChernSchwartz-MacPherson classes, arXiv:math.AG/0202175.

[VV] L. Van Proeyen and W. Veys, Poles of the topological zeta function associated to an ideal in dimension two, Math. Z. (to appear), 17p.

[Ver] J.L. Verdier, Spécialisation de faisceaux et monodromie modérée, Astérisque 101-102 (1983), 332-364.

[Vey1] W. Veys, Relations between numerical data of an embedded resolution, Amer. J. Math. 113 (1991), 573-592.

[Vey2] W. Veys, Poles of Igusa's local zeta function and monodromy, Bull. Soc. Math. France 121 (1993), 545-598. 
[Vey3] W. Veys, Vanishing of principal value integrals on surfaces, J. Reine Angew. Math. 598 (2006), 139-158.

[VZ] W. Veys and W.A. Zuniga-Galindo, Zeta functions for analytic mappings, logprincipalization of ideals, and Newton polyhedra, Trans. Amer. Math. Soc. (to appear), 23p. 